\title{
Numerical modeling of solar wind influences on the dynamics of the high-latitude upper atmosphere
}

\author{
M. Förster ${ }^{1}$, B. E. Prokhorov ${ }^{1,2}$, A. A. Namgaladze ${ }^{3}$, and M. Holschneider ${ }^{2}$ \\ ${ }^{1}$ GFZ German Research Centre for Geosciences, Helmholtz Centre Potsdam, Germany \\ ${ }^{2}$ University Potsdam, Institute for Applied Mathematics, Potsdam, Germany \\ ${ }^{3}$ Murmansk State Technical University, Murmansk, Russia \\ Correspondence to: M. Förster (mfo@gfz-potsdam.de)
}

\begin{abstract}
Neutral thermospheric wind patterns at high latitudes obtained from cross-track acceleration measurements of the CHAMP satellite above both polar regions are used to deduce statistical neutral wind vorticity distributions and were analyzed in their dependence on the Interplanetary Magnetic Field (IMF). The average pattern confirms the large duskside anticyclonic vortex seen in the average wind pattern and reveals a positive (cyclonic) vorticity on the dawnside, which is almost equal in magnitude to the duskside negative one. The IMF dependence of the vorticity pattern resembles the characteristic field-aligned current (FAC) and ionospheric plasma drift pattern known from various statistical studies obtained under the same sorting conditions as, e.g., the EDI Cluster statistical drift pattern. There is evidence for hemispheric differences in the average magnitudes of the statistical patterns both for plasma drift and even more for the neutral wind vorticity. The paper aims at a better understanding of the globally interconnected complex plasma physical and electrodynamic processes of Earth's upper atmosphere by means of first-principle numerical modeling using the Upper Atmosphere Model (UAM). The simulations of, e.g., thermospheric neutral wind and mass density at high latitudes are compared with CHAMP observations for varying IMF conditions. They show an immediate response of the upper atmosphere and its sensitivity to IMF changes in strength and orientation.
\end{abstract}

\section{Introduction}

More than fifty years since the start of the space era with the first satellites, the efforts to understand our space plasma environment and the complexities of its link to solar activities, propagating by the solar wind past the Earth, increase continuously. The dependencies are highly complex and many of them are still not understood in spite of many decades of research with several generations of space missions.

Understanding this complexity is a fundamental problem in physics. This is the subject of space weather science, a relatively new field of research. The term space weather is defined as the conditions on the Sun and in the solar wind, Earths magnetosphere, ionosphere, and thermosphere that can influence the performance and reliability of space-borne and ground-based technological systems and endanger human life or health. As our modern society inexorably increases its dependence on space, the necessity of predicting and mitigating space weather becomes ever more acute (Eastwood, 2008).

This study aims at a better understanding of the coupled magnetosphere-ionosphere-thermosphere (M-I-T) system including its electrodynamics. For the present paper, we confine to investigations of solar wind and IMF forcing processes at the high-latitude upper atmosphere, conveyed by the complex magnetospheric current system and their concomitant electric fields. Beside the dayside thermal heating due to solar EUV and X-ray illumination, the auroral energy sources of particle precipitation and Joule heating, they constitute a main driver for the high latitude upper thermosphere.

The thermospheric mass density and wind have been measured with a relatively new technique, namely an accelerometer as part of the CHAllenging Minisatellite Payload (CHAMP) mission. CHAMP operated throughout the last decade in a near-polar and almost circular orbit near the F-region maximum height. The satellite was developed and managed at the GFZ German Center of Geosciences in Potsdam and turned out to be an exceedingly 


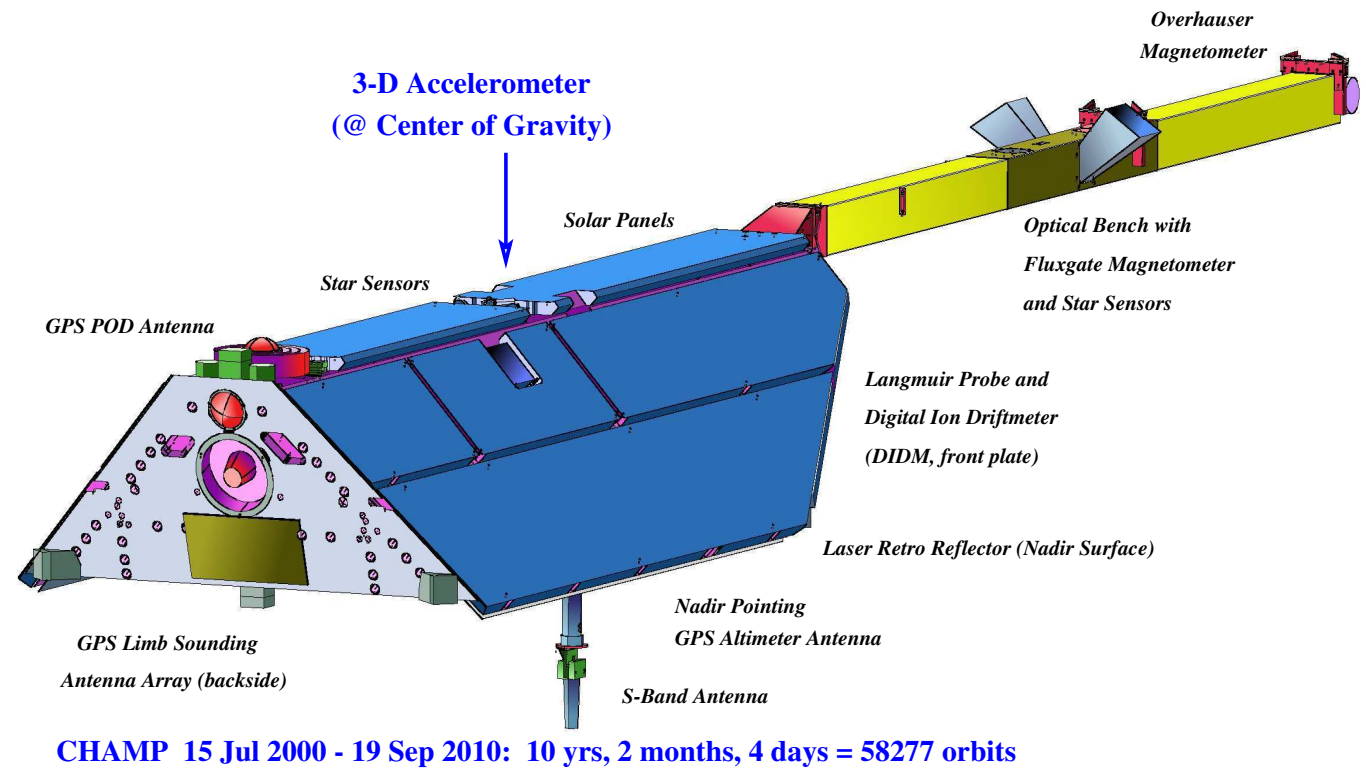

Fig. 1. CHAMP satellite and its equipment (backside view). It wrote a track record of more than one decade in near-Earth orbit.

successful near-Earth geoscience mission. It was launched in summer 2000 into an orbit at $\sim 460 \mathrm{~km}$ with an inclination of $\sim 87.3^{\circ}$ (Reigber et al., 2002) and completed its active measurement lifetime in September 2010 at approximately $260 \mathrm{~km}$ orbit height. It was equipped with a payload suitable for monitoring many parameters relevant for space weather research and application (see Fig. 1). The forthcoming multi-satellite constellation mission Swarm with a similar diagnostics payload will allow even more profound studies of the Earth's magnetic field and the near-Earth environment including space weather aspects and the upper thermosphere dynamics (Friis-Christensen et al., 2006, 2009).

One key scientific instrument onboard CHAMP was the triaxial accelerometer, located at the spacecraft's center of mass. It effectively sampled the in situ acceleration with an accuracy of $\sim 3 \times 10^{-9} \mathrm{~ms}^{-2}$. From the air drag observations, thermospheric mass density and cross-track neutral wind data have been obtained (Doornbos et al., 2010).

First-principle physical-numerical models are nowadays mandatory tools for solving forecasting challenges and for a deeper understanding of the complex interrelated processes in Earth's environment. Modern first-principle numerical models are time dependent, three dimensional (3-D), and global. In this study we will use the Upper Atmosphere Model (UAM) of the Polar Geophysical Institute (PGI) and the Murmansk State Technical University (MSTU).

We had made use of this model already during the preparatory phase of the CHAMP mission as part of the theoretical grounding studies for CHAMP's observational facilities and their applicability for space weather monitoring tasks, as well as for optimal measurement ranges and precision require- ments (Förster et al., 1999a). On that occasion, we studied a moderate storm event and its global-scale implications for neutral air density, composition and wind effects at subauroral to low latitudes as well as the electromagnetic changes with regard to their observability by the instrumentation of CHAMP (Förster et al., 1999b; Namgaladze et al., 2000).

Within the last decade the actual observational outcome of the CHAMP mission is tremendous and the abundance of its data wealthiness is far from being exhausted yet. The CHAMP data provide comprehensive evidences both for influences by solar wind and magnetospheric processes from above (e.g. Förster et al., 2008, 2011) and for upward propagating disturbances from below (e.g. Häusler and Lühr, 2009; Lühr et al., 2011). All these coupling processes are crucial to our understanding of the system's dynamics and variability.

In this study we will focus on global M-I-T coupling effects at high-latitudes. First we present some statistical results of CHAMP's neutral wind accelerometer measurements, which show the systematic dependence of upper thermospheric winds on IMF conditions in the solar wind (Sect. 2). Then we describe the UAM model (Sect. 3) and the specific challenges for modeling the near-Earth space plasma environment and the coupling of magnetospheric processes within the high-latitude ionosphere and thermosphere (Sect. 4). We present our first attempts for systematic analyses of M-I-T coupling processes under various stable IMF conditions (Sect. 5) and conclude the paper with a discussion and an outlook for future modeling efforts (Sect. 6). 


\section{Average neutral wind vorticity at high latitudes}

In the classical M-I-T coupling theory (as reviewed, e.g., by Cowley, 2000), the ionosphere/thermosphere end is treated as a height-integrated boundary of the magnetosphere, where field-aligned current (FAC) systems mediate stress and energy with the magnetosphere-magnetosheath current generation system. It is common view that this coupling occurs by means of ion drag between ionospheric ions set in motion by solar wind induced magnetospheric convection and thermospheric neutrals. Likewise it has long been known that the high-latitude thermospheric wind is stongly influenced by solar wind conditions, in particular by direction and strength of the IMF. This has been shown by satellite missions like Dynamics Explorer (DE-2) back in the early 80-ies already (Hays et al., 1984; Killeen and Roble, 1988; Rees and FullerRowell, 1989; Killeen et al., 1995).

An indirect mechanism of energy transfer from the solar wind to the upper atmosphere was recently brought up by Siscoe and Siebert (2006). It involves the Region-1 FAC system and the geomagnetic field configuration in such a way that the $\boldsymbol{J} \times \boldsymbol{B}$ force acting against the solar wind at the highaltitude end of the Region-1 current loop is transmitted to the Earth as a $\boldsymbol{J} \times \boldsymbol{B}$ force acting on the thermosphere (Siscoe, 2006). Vasyliunas (2007) compares this Lorentz force mechanism as an analog to mechanical leveraging and quotes it the "mechanical advantage" of the magnetosphere in the MI-T system, resulting in an increased thermospheric drag by nearly an order of magnitude over the direct drag mechanism.

The thermospheric wind moves, on the other hand, the conducting ionized layers across the geomagnetic field lines, resulting in a neutral wind dynamo effect that contributes to the overall electrodynamics of the coupled system (Blanc and Richmond, 1980). Moreover, the inertia of the upper atmosphere's motion can help to maintain the ionospheric convection independently of the magnetospheric driver processes which is also known for long time as "fly-wheel effect" of the upper atmosphere (Banks, 1972; Coroniti and Kennel, 1973). The coupled M-I-T system will adjust the various driving processes to result in the plasma convection and thermospheric wind pattern, which we observe.

The complex dynamic response of the coupled processes can be addressd by first-principle global numerical modelings as exemplified later on using the UAM (Sect. 3-5). But first we will show average patterns of the high-latitude upper atmosphere dynamics (Figs. 2-4), in particular its vorticity, which is directly related to these external drivers (see also Förster et al., 2008, 2011). Statistical patterns, like in this study, represent some average behaviour, which might be far from equilibrium conditions within the highly dynamic M-IT system.

The comprehensive CHAMP accelerometer data set and the novel methodology of its data analysis (Doornbos et al., 2010) allow detailed studies of the high-latitude thermospheric wind behaviour at $\mathrm{F}$ region heights. The method to obtain statistically averaged full vector patterns of the high-latitude thermospheric wind from the cross-track (onecomponent) accelerometer measurements has been explained by Förster et al. (2008). In short, the reconstruction of the full horizontal wind vector is done with a Singular Value Decomposition (SVD) method which combines the multiple component measurements of a given bin with their known directions to get the best-fit wind vector estimation for each bin individually. The novelty of our study, based on the well-founded statistics, consists in the first systematic analysis of the thermospheric vorticity in polar regions of both hemispheres in dependence on the IMF orientation.

Figure 2, upper panels, shows the statistically averaged high-latitude horizontal thermospheric wind patterns both for the Northern (left side) and Southern Hemisphere (right) during the years 2002/03 of moderate to high solar activity. We used magnetic coordinates, specifically Altitude Adjusted Corrected Geomagnetic (AACGM) coordinates (e.g., Weimer, 2005, Appendix A), because of the strong geomagnetic control of high-latitude thermospheric dynamics, that has been shown already by early satellite observations (e.g. Hays et al., 1984), and we binned the neutral wind data in the same way as the plasma drift measurements obtained from the Cluster/EDI data over the same time period (Haaland et al., 2007; Förster et al., 2007), in order to make them well comparable. The MLT/magnetic latitude projection of the averaged thermospheric neutral wind vectors is presented here in the same viewing direction for both polar regions, namely such as looking from atop the northern pole downward.

The bottom panels of Fig. 2 present the corresponding neutral wind vorticity, deduced from the circulation patterns by applying Stoke's theorem to each bin, similar to the study of Sofko et al. (1995). It approximates the vorticity $\omega$ across each bin's surface with the integrated flow along the closed path at its boundary to the neighbouring grid cells. Here we define the horizontal wind vorticity with respect to the radial or locally upward direction similar to, e.g., Thayer and Killeen (1991) with positive values (blue) representing cyclonic (counter-clockwise) and negative (red) for anticyclonic (clockwise) rotation. Further we applied spherical harmonic functions (again like in the analogous study for the plasma convection, Haaland et al., 2007) with the same order and degree of the associated Legendre polynomials to represent the vorticity pattern in a similarly smoothed fashion.

The high-latitute neutral wind vorticity in Fig. 2 shows as expected very similar patterns for both the Northern (bottom left) and Southern Hemisphere (bottom right panel). They comprise the well-known large duskside anticyclonic rotation cell with minimum values of $-0.67 \mathrm{mHz}$ and $-0.52 \mathrm{mHz}$, respectively, i.e. with about $25 \%$ larger absolute magnitudes on average at the Northern Hemisphere compared with the Southern Hemisphere. The dawnside cyclonic vorticity is slightly smaller at both hemispheres (about $20 \%$ ) for the overall averages with maximum values of $0.53 \mathrm{mHz}$ 

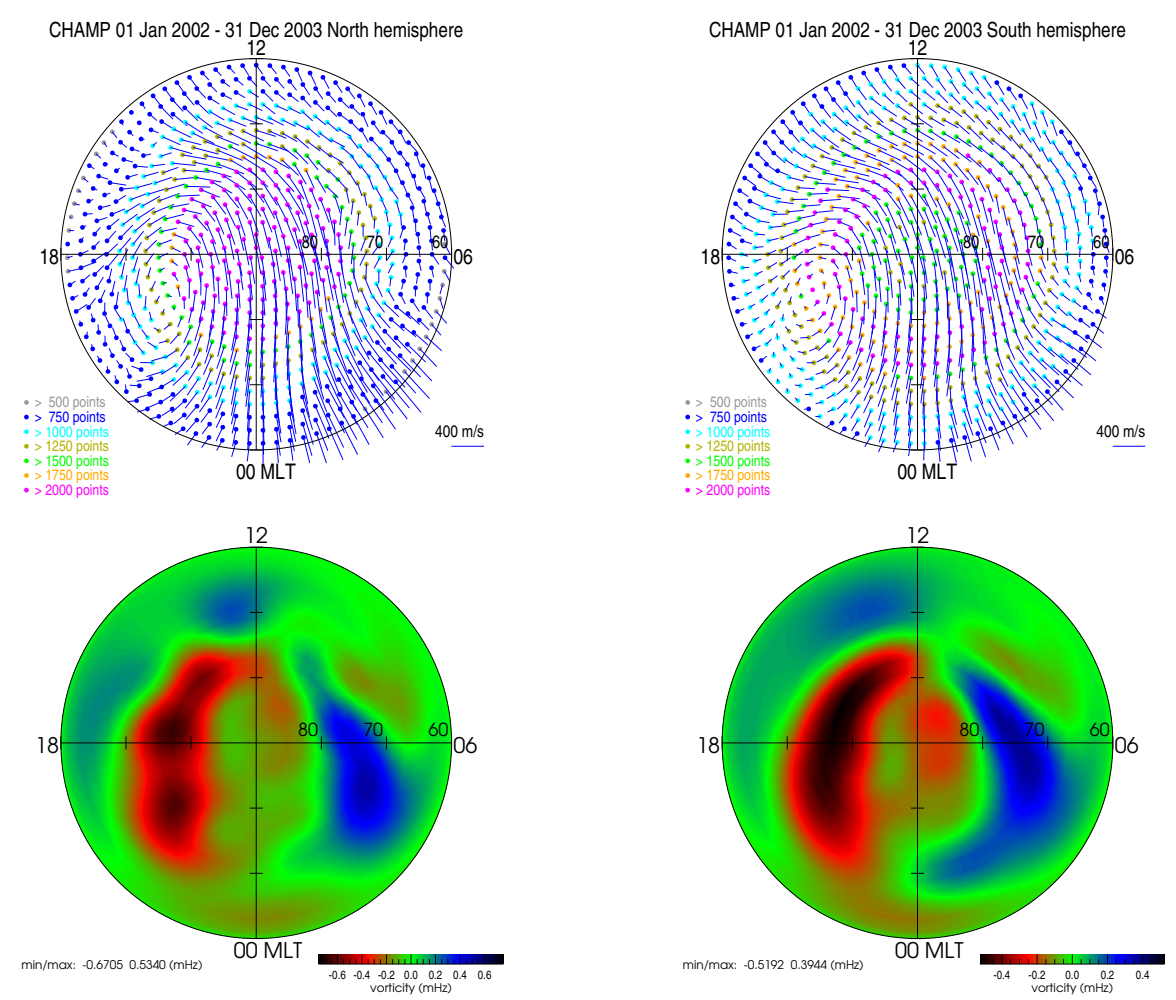

Fig. 2. Average statistic thermospheric wind pattern at $\sim 400 \mathrm{~km}$ height over the Northern (left side panels) and Southern polar hemisphere (right side) obtained from CHAMP accelerometer data of the whole years 2002 and 2003. The MLT versus magnetic latitude dials have an outer boundary of $60^{\circ}$ and sun direction is on top, dusk to the left and dawn to the right side. The neutral wind direction and magnitude are shown in the upper panels by small vectors with the origin in the dots at the bin's position. The bottom panels show the vorticity pattern of the horizontal wind vectors. Negative values (red) indicate a clockwise, positive (blue) a counterclockwise circulation according to the colour bar at the bottom. Minimum and maximum values are indicated there as well (note the different ranges of the colour scales).

Table 1. Minimum and maximum values of high-latitude thermospheric vorticity $[\mathrm{mHz}]$ in both hemispheres at dusk and dawn, respectively, according to the smoothed IMF-dependent patterns in Figs. 3 and 4. The bottom line shows the average behaviour (Fig. 2).

\begin{tabular}{c|ccc|ccc}
\hline IMF & \multicolumn{2}{|c|}{ Northern Hemisphere } & \multicolumn{3}{|c}{ Southern Hemisphere } \\
Sector & $\min$ & $\max$ & $\Delta$ & $\min$ & $\max$ & $\Delta$ \\
\hline 0 & -0.630 & 0.353 & 0.983 & -0.503 & 0.346 & 0.849 \\
1 & -0.661 & 0.445 & 1.106 & -0.664 & 0.342 & 1.006 \\
2 & -0.603 & 0.613 & 1.216 & -0.682 & 0.430 & 1.112 \\
3 & -0.742 & 0.813 & 1.555 & -0.718 & 0.570 & 1.288 \\
4 & -0.917 & 0.700 & 1.617 & -0.579 & 0.562 & 1.141 \\
5 & -0.835 & 0.766 & 1.601 & -0.591 & 0.581 & 1.172 \\
6 & -0.845 & 0.611 & 1.456 & -0.663 & 0.407 & 1.070 \\
7 & -0.735 & 0.409 & 1.144 & -0.626 & 0.426 & 1.052 \\
\hline av. & -0.674 & 0.533 & 1.207 & -0.519 & 0.392 & 0.911 \\
\hline
\end{tabular}

and $0.40 \mathrm{mHz}$, respectively, and shows about the same discrepancy between North and South. The outer border of the vorticity cells follows in shape the statistical equatorward boundary of Region-1 FACs (cf., Förster et al., 2011). The two symmetric crescent-shaped vorticity areas are slightly turned clockwise with respect to the noon-midnight meridian and a view to the wind vector patterns in the upper panels shows their different quality. The duskside vorticity is due to shear motion forming the large dusk vortex, while the dawnside vorticity represents a slight curvature on the large background wind circulation.

Figures 3 and 4 specify the vorticity patterns in dependence on IMF orientation for the Northern and Southern Hemispheres, respectively. The eight sectors represent average neutral wind vorticities for separate IMF ranges, each $45^{\circ}$ wide, around IMF directions indicated on top of each dial. Table 1 summarizes all the minimum and maximum values of the statistic pattern shown in Figs. 3 and 4. Generally, the average vorticity magnitude increases with $B_{z}$ turning southward and becomes largest for southward IMF (sector $4 / 5$ at the Northern, sector 3 at the Southern Hemisphere), while the vorticity magnitudes are smallest and least in latitudinal extent for $B_{z}+$ (sector 0$)$. The dynamic range of the vorticity maxima of the average pattern with respect to the IMF $B_{z}$ component is larger at the Northern Hemisphere.

These IMF-dependencies and their two-cell pattern shapes remind of corresponding patterns for characteristic FAC (cf., 

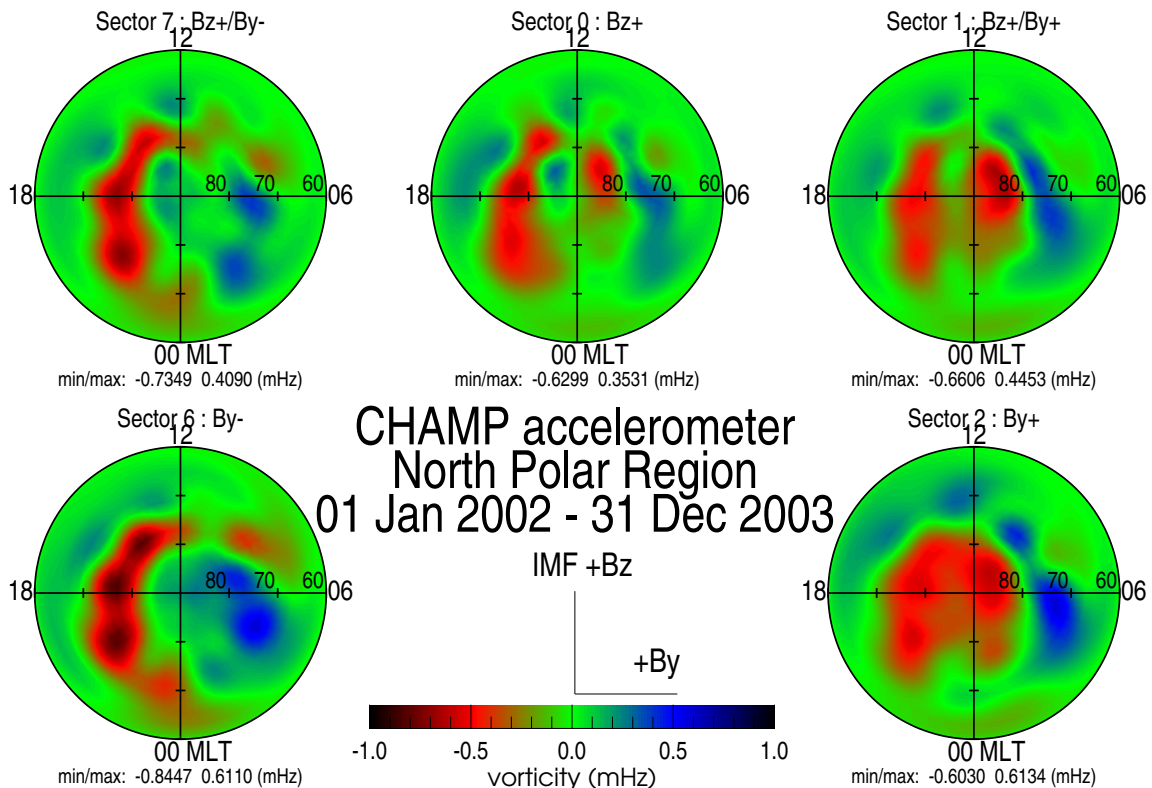

$\min / \max :-0.66060 .4453(\mathrm{mHz})$
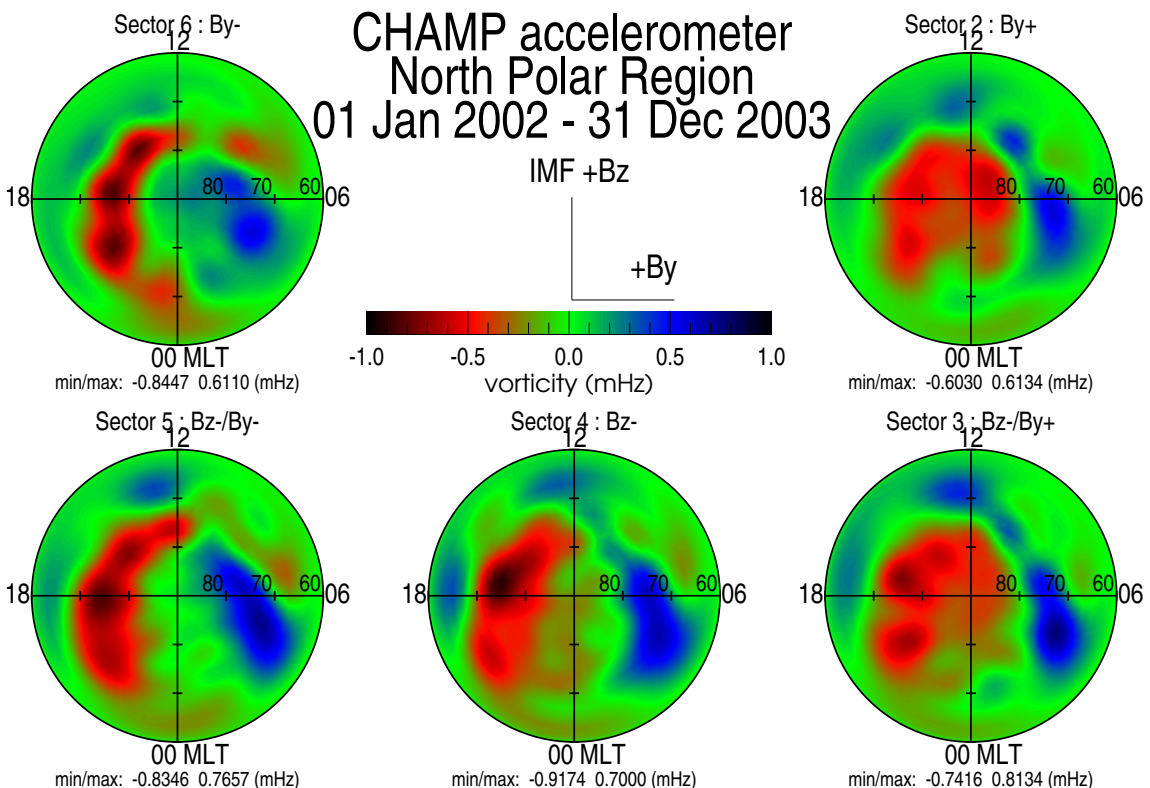

Fig. 3. Average Northern Hemisphere thermospheric vorticity patterns, sorted for 8 distinct sectors of IMF orientation. Each sector comprises $45^{\circ}$ of IMF clock angle range centered around the direction indicated on top of each panel. The data are for the same time interval, presented in the same magnetic latitude/MLT coordinates and colour scale as in Fig. 2, bottom panel. Minimum and maximum values are indicated below each dial (see also Table 1).

e.g., Weimer, 2001; Papitashvili et al., 2002) and plasma drift dependencies on IMF orientation (Ruohoniemi and Greenwald, 2005; Haaland et al., 2007; Förster et al., 2007, 2009). Large circular vorticity cells ("round-shaped") form on the duskside of the Northern Hemisphere for $B_{y}+$ (sectors 1-3), while "crescent-shaped" cells appear there under $B_{y}-$ conditions (sectors 5-7). At the same time the dawnside cell is occupied by "crescent-shaped" or larger "round-shaped" regions, respectively. The patterns are mirror-symmetric with respect to IMF $B_{y}$ for the Southern Hemisphere, where the average magnitudes are generally somehow smaller. For northward IMF (sector 0), there is even an indication of a four-cell structure at high dayside latitudes both at North and South.

\section{The Upper Atmosphere Model (UAM) concept}

The global numerical model of the Earth's upper atmosphere (UAM) has originally been developed at the Kaliningrad Observatory (now West Department) of IZMIRAN (Namgaladze et al., 1988, 1990, 1991). Later it was modified at the Polar Geophysical Institute and the Murmansk State Technical University, in particular for applications at high latitudes (Namgaladze et al., 1996a,b; Volkov and Namgaladze, 1996). The model describes the thermosphere, ionosphere, plasmasphere and inner magnetosphere of the Earth as a single system by means of numerical integration of the corresponding time-dependent three-dimensional continuity, momentum and heat balance equations for neutral, ion and electron gases as well as the equation for the electric field potential. It covers the height range from $60 \mathrm{~km}$ up to 15 Earth radii of geocentric distance, using a fixed dipolar geomagnetic field configuration. The tilt between the geomagnetic and geographic axes of the Earth is taken into account. 


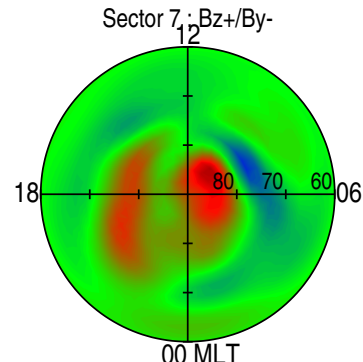

$\min / \mathrm{max}:-0.62630 .4262(\mathrm{mHz})$

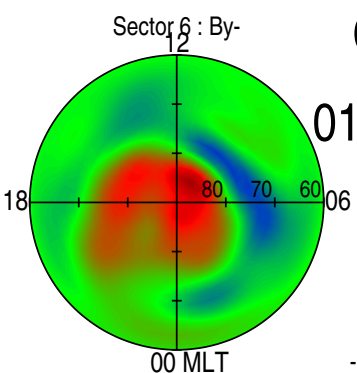

$\min / \max :-0.66270 .4070(\mathrm{mHz})$

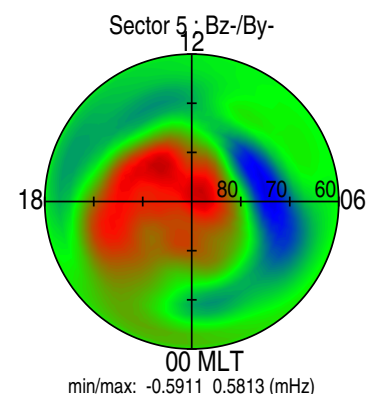

$\min / \max :-0.59110 .5813(\mathrm{mHz})$

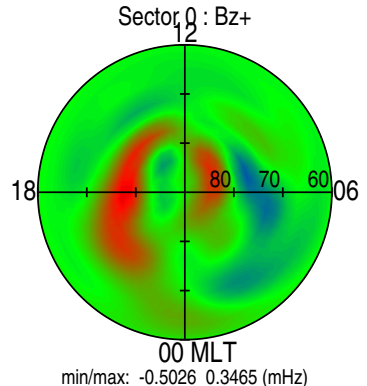

$\min / \max :-0.50260 .3465(\mathrm{mHz})$

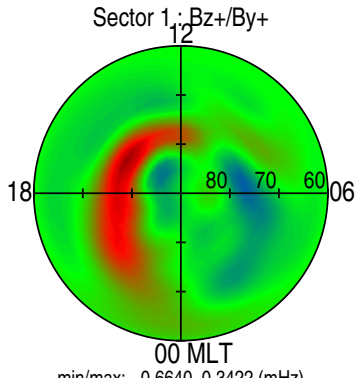

$\min / \max :-0.66400 .3422(\mathrm{mHz})$

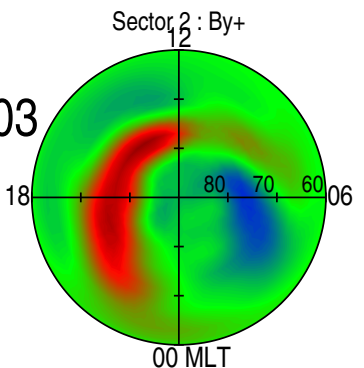

$\min / \max :-0.68170 .4303(\mathrm{mHz})$

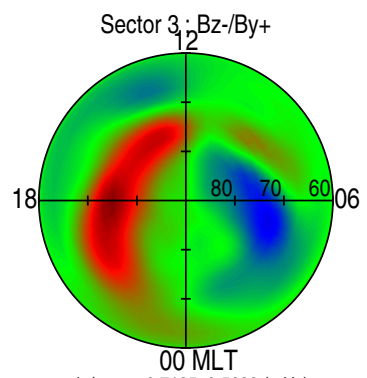

$\min / \max :-0.71850 .5699(\mathrm{mHz})$

Fig. 4. Same as Fig. 3, but for the Southern Hemisphere thermospheric vorticity patterns.

The UAM solves numerically the well-known hydrodynamical continuity, momentum, and heat balance equations for the corresponding constituents by means of finite difference methods (Namgaladze et al., 1988, 1991). It consists of four main blocks (code units) as schematically illustrated in Fig. 5. These blocks describe specific subsets of the complex system in its own appropriate coordinate system as described in detail in previous model descriptions as, e.g., Namgaladze et al. (1996b, 1998c, 2000). The magnetospheric block, in particular, has been incorporated into the model in the 90ies (Volkov and Namgaladze, 1996). This block calculates the density, pressure and drift velocity of the hot ions in the magnetospheric plasma sheet as well as the Region-2 FACs. The exchange of information between the blocks is realized by interpolation schemes from the grid points of one coordinate system to the other, while the solutions are found in an iterative manner. Improved versions of the model (Namgaladze et al., 1996b, 1998a,b) allow variable grid schemes with higher resolutions in certain regions as, e.g. the auroral and cusp zones, in dependence of the physical problem to be solved. The time steps have to be adopted accordingly to avoid undesirable numerical effects (Ridley et al., 2010).

The time-dependent transport equations of the model are second-order parabolic ones; the model formulation has to comprise therefore both initial and boundary conditions for all the constituents. An inherent part of the model formulation is also the state-of-the-art compilation of a multitude of transport coefficients, cross sections, chemical reaction rates, heating efficiencies etc., which can have a devastating effect on the results, if they are not properly chosen.

Model inputs are (1) the solar UV and EUV spectra, (2) the precipitating particle fluxes (electrons and energetic ions), and (3) the cross-polar cap potential drop $\left(\Delta \Phi_{\mathrm{PC}}\right)$ or, alternatively, suitable Region-1 (and, when indicated, Region-0) FACs, which represent the primary external drivers for the M-I-T system (reconnection processes).

\section{Model challenges (open questions)}

The necessary input values, mentioned at the end of the previous section, require the use of empirical models. Such 


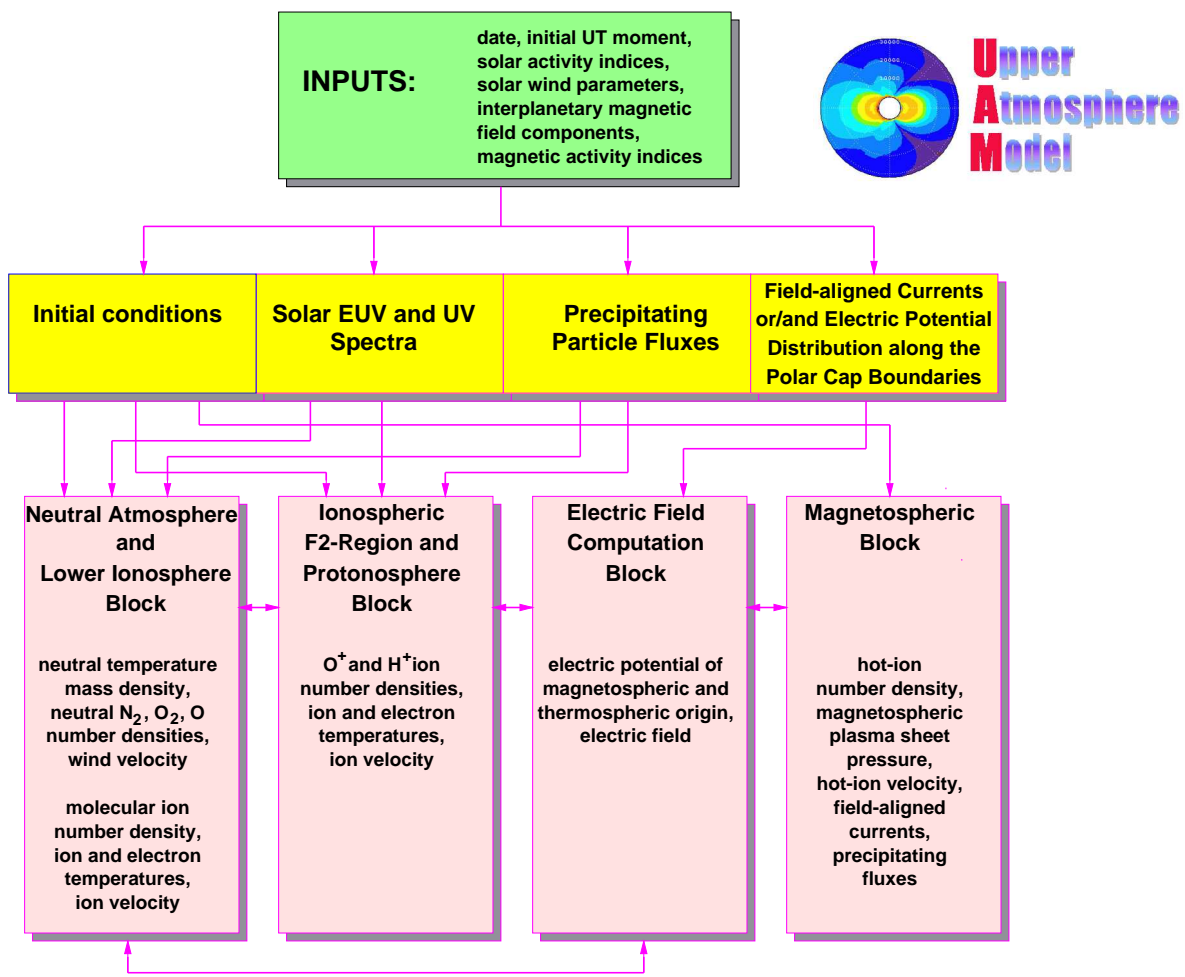

Fig. 5. Schematic structure of the Upper Atmosphere Model (UAM Namgaladze et al., 1988, 1991, 1996b, 1998a,b) with its inputs (initial and boundary conditions), the four main computational blocks, and their output parameters (taken from Förster et al., 1999a, Fig. 3).

Table 2. Selected time intervals with extended stable IMF conditions.

\begin{tabular}{|c|c|c|c|c|c|c|c|c|c|c|c|c|c|c|c|c|}
\hline \multirow[t]{2}{*}{ Date } & \multirow{2}{*}{$\begin{array}{l}\text { timing } \\
\text { UT, h }\end{array}$} & \multirow{2}{*}{$\begin{array}{l}\text { MJD } \\
2000\end{array}$} & \multirow{2}{*}{$\begin{array}{c}\text { IMF } \\
\text { Sector }\end{array}$} & \multicolumn{4}{|c|}{ Average IMF values [nT] } & \multicolumn{8}{|c|}{ 3-hourly } & \multirow{2}{*}{$\begin{array}{l}\text { Daily } \\
\text { F10.7 }\end{array}$} \\
\hline & & & & $|B|$ & Bx & By & $\mathrm{Bz}$ & & & & Kp & lices & & & & \\
\hline 14 Apr 2001 & $15-11^{*}$ & 469 & 6 & 7.7 & -2.5 & -7.2 & -1.3 & $4+$ & $4-$ & $3+$ & $3+$ & $3+$ & $3+$ & $3-$ & 20 & 181.0 \\
\hline 25 May 2002 & $18-12 *$ & 875 & 1 & 9.8 & -6.3 & 6.3 & 4.0 & $1-$ & $0+$ & $0+$ & $0+$ & $1+$ & 20 & $1+$ & 10 & 187.4 \\
\hline 18 Nov 2002 & $00-24$ & 1052 & 5 & 8.8 & 2.1 & -6.4 & -5.6 & 30 & 20 & $2-$ & $1+$ & $2+$ & 20 & 30 & 30 & 174.8 \\
\hline $01 \mathrm{Feb} 2003$ & $14-14^{*}$ & 1127 & 6 & 9.8 & 3.2 & -9.2 & -1.2 & 30 & 20 & $1-$ & $1-$ & $1+$ & $3-$ & $5-$ & $4+$ & 122.1 \\
\hline 23 Oct 2003 & $00-21$ & 1391 & 0 & 7.0 & -1.9 & -0.6 & 6.7 & $2-$ & $3+$ & $3-$ & $3-$ & 30 & $4-$ & 30 & 40 & 141.7 \\
\hline 30 Aug 2004 & $08-22$ & 1703 & 4 & 12.6 & 6.8 & 3.0 & -10.2 & $2+$ & 20 & $3+$ & 40 & 40 & $6-$ & 50 & 70 & 91.6 \\
\hline 13 Sep 2004 & $22-21^{*}$ & 1717 & 6 & 10.6 & 6.0 & -8.4 & 2.2 & 0o & 0o & 0o & $0+$ & 0o & $0+$ & $4+$ & $4+$ & 119.1 \\
\hline 06 Oct 2004 & $13-22$ & 1740 & 2 & 4.1 & 0.1 & 4.0 & -1.0 & 10 & $2-$ & $1+$ & 10 & 0o & $0 \mathrm{o}$ & $0+$ & $0+$ & 92.0 \\
\hline 10 Dec 2004 & 09-21 & 1805 & 2 & 8.1 & -1.0 & 7.6 & -2.4 & 20 & $2+$ & 20 & 20 & $2+$ & 30 & $2+$ & 30 & 82.3 \\
\hline 13 Jun 2005 & 00-14 & 1990 & 3 & 12.2 & -1.9 & 9.8 & -6.9 & 60 & $6-$ & $4+$ & 30 & 40 & $3-$ & $3+$ & $2-$ & 94.7 \\
\hline 30 Apr 2006 & $10-24$ & 2311 & 2 & 3.4 & -3.2 & 1.1 & 0.5 & 0o & 0o & $0 \mathrm{o}$ & $0 \mathrm{o}$ & 0o & 0o & 0o & 0o & 101.4 \\
\hline 02 Nov 2006 & $21-08^{*}$ & 2497 & 2 & 5.5 & 3.5 & 4.0 & -1.3 & $2+$ & 20 & 10 & 10 & $2-$ & $3-$ & 30 & $2+$ & 86.8 \\
\hline
\end{tabular}

* Interval starts at the previous day already.

models usually describe the magnitude, spatial distributions and temporal variations of certain input values in dependence of either solar, IMF parameters or geomagnetic indices. These models are as a rule generalizations of large observational data sets and describe often so-called climatological variations, but not necessarily the actual situation.

To overcome this difficulty and to skip the "ditch" between generalized or "climatological" modeling and actual simulations of real situations, one has to seek for as much as possible real, actual measurements for all kind of input values from solar EUV fluxes, electrodynamic parameters, till high-energy precipitations. Any real observation of the environmental plasma situation, which can be acquired for comparison, can therefore improve the modeling performance by constraining or optimizing the possible solutions. 
In this study we are concerned with complex processes at high-latitudes, which constitute particularly interesting matters for the externally driven M-I-T interlinking. The correct matching of the various inputs (fluxes, FACs, boundary positions) within relatively small distances (which might even reach to sub-grid dimensions) is here of particular importance. In contrast to global studies, which focus on subauroral to mid- and low latitudes (like, e.g., the study of Förster et al., 1999b; Namgaladze et al., 2000), where the high-latitude energy input can be handled as a far-distance standard-shaped oval source, we have to treat these inputs very carefully within and near the auroral oval.

Such input values are for instance the solar EUV and $\mathrm{x}$-ray spectra with newly developed solar indices (Tobiska et al., 2006) and electron precipitation fluxes given by Hardy et al. (1985) and their latitudinal and longitudinal distribution. Most of such models are parametrized with respect to solar or global geomagnetic indices like the $F_{10.7}$ proxy of solar EUV flux intensities and the three-hourly Kp values. Somehow exceptional are models that describe environmental conditions with respect to solar IMF strength and orientation. They exist for such input quantities like the ionospheric convection electric field (Ruohoniemi and Greenwald, 2005; Haaland et al., 2007; Förster et al., 2009) and FAC distributions (Weimer, 2001; Papitashvili et al., 2002).

Any misfit between input models can have tremendous consequences for UAM results of, e.g., the electric field pattern and therefore also for the plasma distribution and neutral wind pattern. It is well known that the electric field and current distributions depend strongly on the ionospheric conductance (Ridley et al., 2004). This concerns in particular the current carrying layers, which are formed at auroral latitudes primarily by energetic particle precipitations. But also the terminator position plays a role, which depends on season (Ridley, 2007), but also on UT (longitude) due to the tilt between the geomagnetic and geographic axes for a dipolar field configuration like in the UAM (the real geomagnetic field with differences of the configurations between the hemispheres can complicate the situation even more).

For this model study with its focus on M-I-T coupling processes in dependence of the IMF strength and orientation, we used the FAC model of Papitashvili et al. (2002), including its extended auxiliary material. The idea was to have an external driver representation, which comprises explicitely the IMF dependencies. Alternatively, we could use also IMFdependent models of the magnetospheric drift pattern like that of Förster et al. (2009) or the ground-based SuperDARN observations of the ionospheric drift pattern as summarized by Ruohoniemi and Greenwald (2005). All these models represent parametrized statistical averages, i.e. they show smoothed, quasi-static patterns in a "climatological" sense. They show generally smaller amplitudes as a multitude of real distribution patterns, all the second-order dependencies and the natural variabilities are "smeared" into spherical harmonic functions of finite order and degree.
The FAC model of Papitashvili et al. (2002) was derived from high precision magnetic field measurements from the Ørsted and Magsat satellites, being parameterized by the IMF strength and direction for summer, winter, and equinox as well as explicitely for both polar regions separately, resolving the seasonal dependence of interhemispheric asymmetries. The FAC distributions are fitted with associated Legendre functions, limited to degree $n=21$ and order $m=3$ to obtain the FAC patterns with the fewest terms, and continued downward to the ionospheric altitude of $115 \mathrm{~km}$ using current conservation (Papitashvili et al., 2002).

First UAM simulation attempts with these patterns revealed promptly that the resulting drift pattern and the crosspolar cap potential (CPCP) show unrealistic small values, obviously due to the outspread FAC distributions of the spherical harmonics. We therefore repeated the experiments with a modified FAC distribution, which limits the actual FAC belts in latitude ranges corresponding to the most likely auroral oval positions, keeping the main characteristics of the FAC model patterns with its IMF and seasonal dependence. This was done by integrating the model current densities along each meridional cut and assigning these values to the belt's position, both for Region-1 and Region-0 (within the auroral oval) FACs. The oval position, including $B_{y}^{\mathrm{IMF}}$-dependent turning and $B_{z}^{\mathrm{IMF}}$-dependent widening or shrinking, has to be harmonized with other model's boundary positions like, e.g., the open-closed boundary (OCB) and the precipitation patterns. The cusp position is of particular importance because of its crucial role in the M-I-T dynamics as observed by CHAMP (e.g., Schlegel et al., 2005; Rentz and Lühr, 2008) and modeled with UAM (Namgaladze et al., 1996b, 1998c).

The choice of the suitable coordinate system is an important issue for a meaningful model-observation comparison, in particular at high-latitudes. The model operates with a tilted dipol magnetic field constellation, while most of empirical models for the high-latitude parameters and the observations of CHAMP are provided in corrected geomagnetic coordinates (or even AACGM for a correct mapping of data along geomagnetic field lines). While the model results in the subsequent presentations (Figs. 8-9) are plotted in geomagnetic coordinates (MLT versus geomagnetic latitude), the overplotted CHAMP orbital track and the measurements are shown according to their AACGM coordinates. We think that comparisons come hence as close as possible to reality.

A slightly different, but related question is the correct positioning of the various high-latitude boundaries within the dipolar model formulation and how well it can reflect the observations. In future, it might be necessary to replace UAM realizations of the geomagnetic field configuration at least for the electric field computation and the magnetospheric block - by more realistic approximations of the actual field, as it is supplied, e.g., by the Tsyganenko model of the magnetosphere (Tsyganenko, 2002a,b). This model 

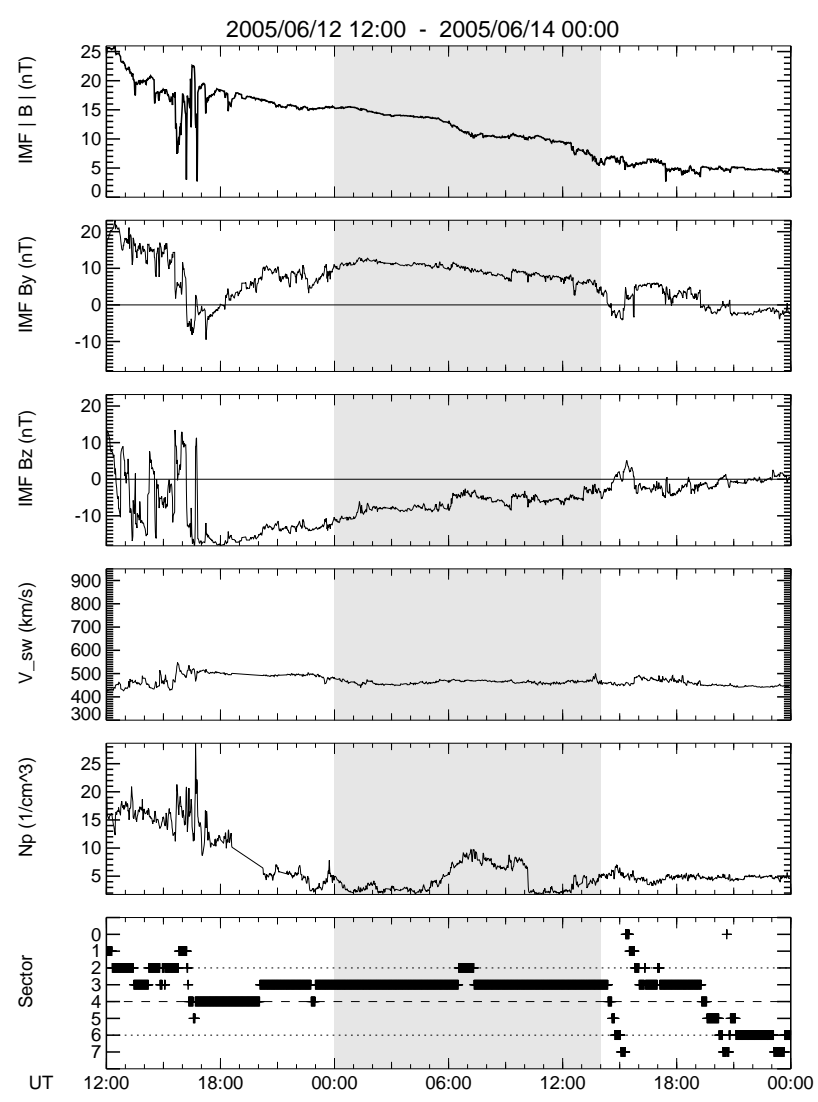

Fig. 6. Solar wind and IMF conditions during one of the intervals with stable IMF conditions (shaded, \# 10 according to Table 2), obtained from ACE observations at the Earth-sun L1 libration point upstream of Earth and time-shifted to the magnetopause position according to a procedure, which is described in detail in Haaland et al. (2007). From top to bottom, the panels show the IMF magnitude, the IMF y- and z-components in GSM coordinates, the solar wind velocity, the solar wind plasma density, and the corresponding sector of the IMF in the GSM-y-z plane (see text).

is also parametrized with respect to solar wind and IMF conditions.

\section{First comparisons for stable IMF conditions}

Magnetospheric behaviour at any given time moment depends strongly on the actual forcings and its pre-history. In other words, the M-I-T system is very dynamic and highly time-dependent. This is reproduced by the UAM as a timedependent 3-D realization with initial and boundary conditions. The temporal development constitutes a key question for realistic modeling, in particular for storm events. As we focus in this study on IMF dependencies and hemispheric differences, we primarily tested the UAM performance under relatively stable IMF conditions. As listed in Table 2, we selected a series of time intervals of the CHAMP lifetime with various IMF conditions, each of which persisted for longer time intervals of at least several hours. It is supposed that the M-I-T system then attains some kind of dynamic equilibrium state that can more easily be compared with observations.

Figure 6 shows the solar wind and IMF conditions around one of the intervals with (more or less) stable IMF conditions, namely the 10th of the 12 listed in Table 2 . The IMF values obtained by the Advanced Composition Explorer (ACE) spacecraft, are time shifted to the frontside magnetopause (at $\mathrm{X}_{\mathrm{GSM}}=10 \mathrm{R}_{E}$ ), using the phase front propagation technique of Weimer et al. (2003) in a slightly modified version, which is based on constrained minimum variance analyses of the IMF (Haaland et al., 2006). The procedure of sorting for specified IMF directions (clock angle sectors) and the bias value filtering of solar wind data for stable IMF conditions has been described in the companion papers of Haaland et al. (2007) and Förster et al. (2007). Here, we use the same methodology with the same bias value $(\geq 0.96)$ threshold as for these Cluster/EDI plasma convection analyses and previous high-latitude neutral wind studies (Förster et al., 2008, 2011).

The grey-shaded period in Fig. 6 is supposed to be sufficiently stable - in this case for about $14 \mathrm{~h}$. This interval is insofar exceptional, as it proceeds during the recovery phase of a moderate storm that occured during the preceding day. The geomagnetic indices Dst, AE, and Kp in Fig. 7 illustrate this situation. The 3-hourly Kp values are still at a high level during the begin of the interval and relax slowly, while the $\mathrm{AE}$ values fluctuate around $900 \mathrm{nT}$ indicating considerable auroral activity. The IMF orientation is stable with considerable $B_{z}-$ and $B_{y}+$ values corresponding to sector 3 conditions, while the magnitude $\left|B^{\mathrm{IMF}}\right|$ declines steadily during the interval.

Fig. 8 shows UAM simulation results for high-latitude circumpolar regions in geomagnetic coordinates with $\left|\phi_{m}\right|>$ $60^{\circ}$ for both the Northern (left panel) and Southern (right) Hemisphere together with CHAMP cross-track neutral wind measurements along two subsequent passages at almost $\sim 400 \mathrm{~km}$ altitude over the respective polar regions. The modeled parameters shown are the electric potential in the background with the black-white scale and the horizontal neutral wind field represented by coloured arrows with the scale on top. The model results refer to an altitude level that is indicated in the lower right corner; it corresponds to the satellite height at the moment indicated by the black arrow and written in the middle of the bottom line. Minimum and maximum values along the CHAMP orbital section are shown in the lower left corner of each panel both for the observed and the modeled values.

During this time interval, CHAMP crosses the large dusk vortex and the dawn cell approximately in the dawn-dusk plane. A large "round-shaped" dusk convection cell and simultaneously a large dusk side neutral wind vortex can be noticed at the Northern Hemisphere (Fig. 8, left panel) with maximum wind speeds across the polar cap region on the 

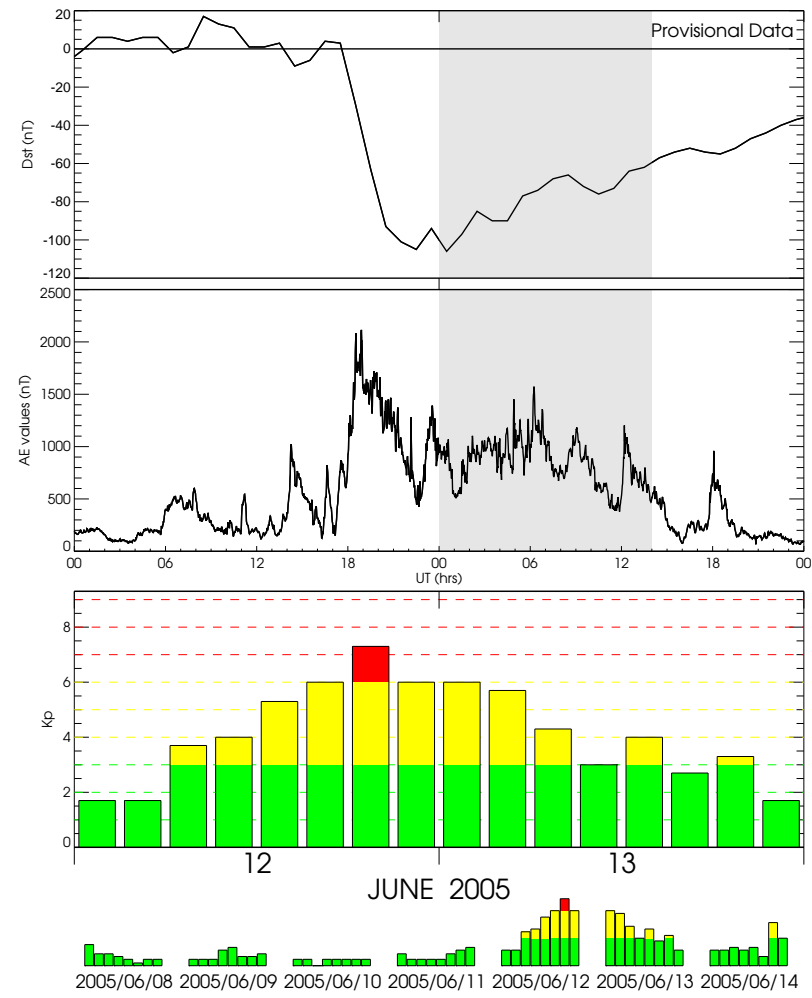

Fig. 7. Geomagnetic conditions during the same interval (\# 10 of Table 2) as in Fig. 6 with stable IMF conditions (shaded). The panels from top to bottom show the hourly Dst index, the one-minute $\mathrm{AE}$ values, and the 3-hourly $\mathrm{Kp}$ index for the current and the encircling days. The interval appears to happen during a minor storm recovery phase.

dawnward side. This is in accord with the statistical average pattern of sector 3 IMF orientation both for plasma convection (cf. Fig. 7 of Haaland et al. (2007)) and the neutral horizontal wind (Fig. 3 above).

The modeled neutral wind component in the cross-track direction along the satellite's orbit agrees closely with the observed wind profile. The position of the dusk vortex center, as it is accidentially almost centrally crossed by the satellite, seems to be closely reproduced as it is indicated by the change of the wind direction from sunward in the subauroral region $\left(<70^{\circ}\right)$ to anti-sunward at the poleward side. The modeled wind velocity shows smooth variations in contrast to the observations, which reveal superposed smaller-scale minor variations.

The plasma convection and neutral wind circulation pattern during the crossing over the Southern polar region (Fig. 8, right panel) shows a more symmetric two-cell pattern with largest wind velocities in antisolar direction over the central polar cap. The dusk vortex center is shifted to lower latitudes and to slightly earlier local time hours ( $\sim 17$ MLT). The dusk vortex shrinked in its latitudinal extent, so that both vortices have now about the same size. This corresponds to the statistically expected pattern for Sect. 3 IMF conditions at the Southern Hemisphere (cf. Fig. 8 of Haaland et al. (2007) and Fig. 4, respectively).

The modeled neutral wind profile along the orbit reproduces the latitudinal variation, but the amplitude does not everywhere match the observations. It differs on the duskward side inside the polar cap region up to about a factor of two, while it coincides in the region of the dawnside vortex. The maximum wind amplitude of the CHAMP observations of more than $900 \mathrm{~m} \mathrm{~s}^{-1}$ in this case appears to be quite large.

Figure 9 shows UAM-CHAMP comparisons for the neutral mass density in the same manner and for the same time moments as in Fig. 8. Here, the modeled parameter is shown in the background with the colour scale on top and the minimum/maximum values of the model area indicated just below the scale on its begin and end. The corresponding $\mathrm{min} / \mathrm{max}$ values of both CHAMP and UAM values along the orbital trace are written in the lower left corner of each panel.

The agreement between model and observation for the Northern traverse of the polar region (Fig. 9, left panel) is good, except for two mass density peaks on the dawn side. They probably indicate the position of the cusp in the prenoon sector near $\sim 74^{\circ}$ and 08:30 MLT (cf. Schlegel et al., $2005)$ and an other auroral feature at lower latitudes $\left(\sim 66^{\circ}\right)$.

The mass density profile of the CHAMP observations over the Southern Hemisphere (Fig. 9, right panel) clearly differs from the modeled ones both in amplitude and latitudinal trend. This misfit over the southern winter polar region is not seen at the beginning of this stable IMF interval (not shown), but started around 03:00 UT and continued with a steady increase toward the interval's end for unclear reasons. At the Northern Hemisphere the density differences between model results and observations starts later on this day ( $\sim 12: 00$ UT), is less distinctive and does not show differences in trend. There is obviously some thermospheric heating process, which is not yet correctly reproduced by the UAM modeling.

\section{Discussion and conclusions}

This paper presents statistical neutral wind vorticity patterns based on accelerometer data of the CHAMP satellite. It shows the strong IMF dependence of the high-latitude upper atmosphere neutral wind at F-region heights $(\sim 400 \mathrm{~km})$. These patterns confirm previous findings based on early satellite data in the 1980s (e.g., Killeen and Roble, 1988; Killeen et al., 1995), but can now rest upon a much broader statistical base due to the good coverage of the CHAMP data set (cf. also Förster et al., 2011).

Averaging over seasonal effects, these patterns provide an observational evidence for hemispheric differences in the amplitudes of the neutral wind vorticity, which amounts in 

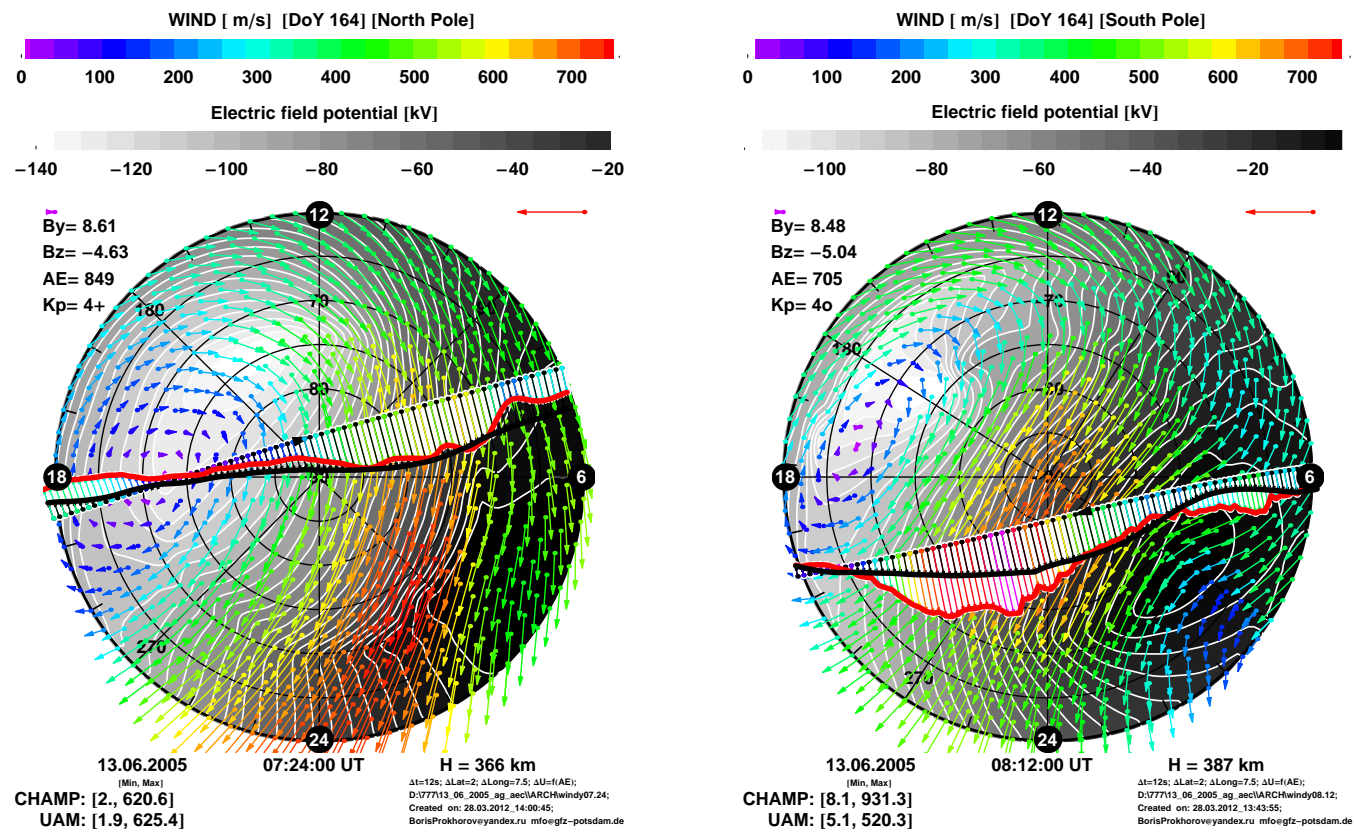

Fig. 8. UAM model results compared with cross-track accelerometer measurements of the CHAMP satellite for overflights over the Northern (left panel) and Southern Hemispheres (right panel) in polar geomagnetic coordinates at latitudes higher than $60^{\circ}$ during the interval \# 10 according to Table 2 (see also Figs. 6 and 7 for solar and geophysical conditions). The dials show sun direction (12 MLT) on top, dusk side (18 MLT) to the left and dawn (06 MLT) to the right, respectively. Magnetic longitude coordinates are indicated with the black crosslines. The dotted line follows the orbital track from $\sim 18$ MLT to $\sim 08$ MLT at North (left) and from $\sim 20$ MLT to $\sim 06$ MLT at South (right panel) with larger arrows at the actual spacecraft positions at 07:24 UT and 08:12 UT, respectively. The cross-track fine black arrows correspond to the calculated UAM crosswind speed, while the colour arrows show cross-track wind component measurements of CHAMP. The model wind vector pattern (coloured arrows according to the scale on top) and the electric potential (b/w colour scale) are shown in the background.

the statistical average to about $25 \%$ - with larger values at the Northern Hemisphere compared to the Southern. A small difference between North and South in the statistical sense was already found for the plasma drift pattern (or CPCP) with $\sim 7 \%$ larger average drift velocities at the Northern Hemisphere compared to the Southern (Förster et al., 2007).

These differences are most likely due to the different geographic-geomagnetic offsets between the hemispheres (Förster et al., 2008). Alternatively, it might be conceivable that the differences of statistical averages could be ascribed to unequal weighting effects due to seasonal or UT dependent conductance differences between the hemispheres. Finally, they could even be due to different patterns of geomagnetic flux densities at the opposite polar regions, i.e. due to higher order geomagnetic field components, which are known to exist to an appreciable extent. Further UAM modeling studies should help to clarify this question. In this regard it will be interesting, whether the present symmetric model configuration with the tilted dipolar field is sufficient to explain the hemispheric differences or whether a more realistic geomagnetic field configuration has to be invoked.

It was noted already in earlier studies that the anticyclonic dusk side thermospheric neutral wind vortex is almost invari- ably stronger than the cyclonic vortex associated with the dawn side ion convection cell (Killeen and Roble, 1988). These observations were accompanied by comprehensive theoretical-numerical studies (e.g. Rees and Fuller-Rowell, 1989), which ascribe this difference to the effects of the Coriolis and centrifugal forces. They tend to maintain the dusk side anticyclonic vorticity, while partly compensating each other on the dawn side.

A previous CHAMP study by Lühr et al. (2007) on average high-latitude neutral thermospheric wind pattern came, bye the way, to different results than those shown in Fig. 2. They obtained distributions of mean thermospheric wind vectors in both polar regions by means of a minimum variance technique (Fig. 4 and equ. 4 of Lühr et al., 2007, respectively). Because of the sometimes shallow minima, they guess that the correct wind direction could also be obtained from the maximum values of that function, when turning it by $90^{\circ}$. This methodological error results in different wind pattern, mainly in the early morning and afternoon sectors.

The variation of the vorticity pattern with IMF $B_{y}$ and $B_{z}$ (Fig. 3 and 4) strongly resembles the corresponding pattern of the plasma drift (e.g., Ruohoniemi and Greenwald, 2005; Haaland et al., 2007) and FACs (e.g., Papitashvili et al., 

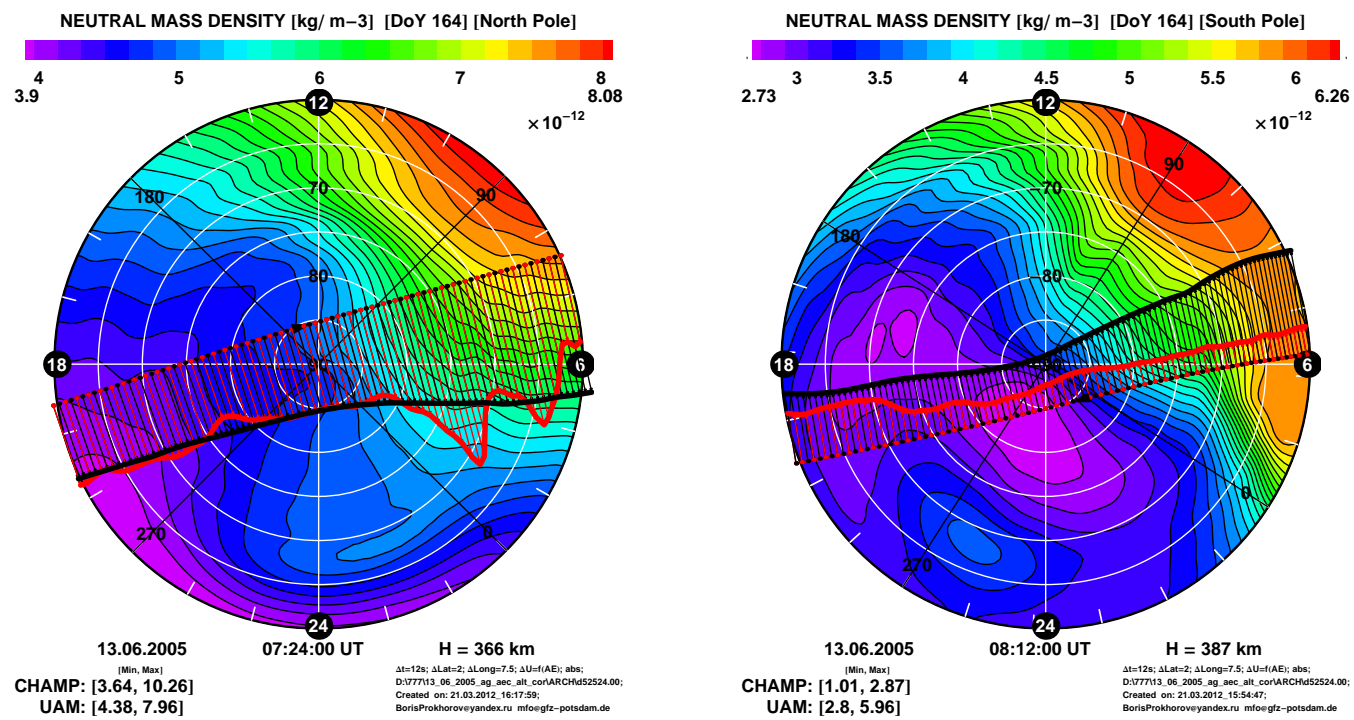

Fig. 9. UAM model results for the same interval and in a similar fashion like in Fig. 8, but here compared with CHAMP accelerometer mass density measurements for overflights over the Northern (left panel) and Southern Hemispheres (right panel) in polar geomagnetic coordinates at latitudes higher than $60^{\circ}$. The absolute mass density values along the orbital track are shown as cross-track distances with dashed black lines for the UAM model results and red coloured ones for the CHAMP observations. Mimimum and maximum values along this orbital section are listed in the left bottom corner both for CHAMP and UAM model data (in units of the top colour scale). The mass density pattern at the height level of the satellite as indicated in the bottom line is shown in the background with the colour scale at the top.

2002). Additional to the strong changes of extent, shape and position of the dusk and dawn circulation cells with the IMF orientation, there is also a systematic variation of their maximum amplitudes. They are largest for sector 3 at South and for sector $4 / 5$ at the North Hemisphere. This comes along with the largest cross-polar thermospheric wind amplitudes over the central polar cap for these IMF orientations at the respective hemisphere, as shown by Förster et al. (2008).

Statistical patterns of high-latitude magnetic field-aligned plasma vorticity with the same sorting for 8 sectors of IMF orientation have been deduced recently also from SuperDARN measurements (Chisham et al., 2009). They show the close affinity to corresponding FAC pattern, despite of smaller variations due to seasonal (ionospheric conductance) effects. In a subsequent study, Chisham and Freeman (2010) investigated the probability density function of plasma vorticity measurements and showed that it is best modeled by the q-exponential distribution across most of the polar ionosphere, except in the dayside Region-1 current region, where the Weibull distribution provides the best model. They interpret this as an indication for the relative importance of different physical mechanisms affecting the plasma drift vorticity in the various regions with a dominance of convective (barotropic) effects within the dayside Region-1 and baroclinic vorticity elsewhere (Chisham and Freeman, 2010). The clear-cut neutral wind vorticity regions and their obvious IMF dependence indicate a strong relevance of the neutral wind for this plasma vorticity behaviour.
For a deeper understanding of the M-I-T system, adequate numerical models are indispensable due to the complexity and nonlinearity of the inherent coupling processes. This concerns first of all global, time-dependent first-principal MHD models like the UAM in this study. We presented our first attempts for an adequate modeling of the high-latitude CHAMP measurements to obtain new physical insights into complex M-I-T processes. The forthcoming Swarm satellite mission will allow much more detailed studies of the high-latitude thermosphere dynamics and its complex interaction with magnetospheric configurations under varying solar wind conditions.

Acknowledgements. Work at GFZ German Research Centre for Geosciences Potsdam and the University Potsdam was supported by Deutsche Forschungsgemeinschaft (DFG). We thank the ACE SWEPAM and MAG instrument teams and the ACE Science Center for providing the ACE data. The CHAMP mission is sponsored by the Space Agency of the German Aerospace Center (DLR) through funds of the Federal Ministry of Economics and Technology, following a decision of the German Federal Parliament (grant code 50EE0944). The data retrieval and operation of the CHAMP satellite by the German Space Operations Center (GSOC) of DLR is acknowledged. 


\section{References}

Banks, P. M.: Magnetospheric processes and the behavior of the neutral atmosphere, Space Res., 12, 1051-1067, 1972.

Blanc, M. and Richmond, A. D.: The ionospheric disturbance dynamo, J. Geophys. Res., 85, 1669-1686, 1980.

Chisham, G. and Freeman, M. P.: On the non-Gaussian nature of ionospheric vorticity, Geophys. Res. Lett., 36, L12103, doi:10. 1029/2010GL043714, 2010.

Chisham, G., Freeman, M. P., Abel, G. A., Bristow, W. A., Marchaudon, A., Ruohoniemi, J. M., and Sofko, G. J.: Spatial distribution of average vorticity in the high-latitude ionosphere and its variation with interplanetary magnetic field direction and season, J. Geophys. Res., 114, A09301, doi:10.1029/2009JA014263, 2009.

Coroniti, F. V. and Kennel, C. F.: Can the ionosphere regulate magnetospheric convection?, J. Geophys. Res., 78, 2837-2851, 1973.

Cowley, S. W. H.: Magnetosphere-Ionosphere Interactions: A Tutorial Review, in: Magnetospheric Current Systems, edited by: Ohtani, S.-I., Fujii, R., Hesse, M., and Lysak, R. L., 118, Geophysical Monograph, 91-106, American Geophysical Union, 2000.

Doornbos, E., van den IJssel, J., Lühr, H., Förster, M., and Koppenwallner, G.: Neutral density and crosswind determination from arbitrarily oriented multi-axis accelerometers on satellites, J. Spacecraft Rockets, 47, 580-589, doi:10.2514/1.48114, 2010.

Eastwood, J. P.: The science of space weather, Phil. Trans. R. Soc. A, 366, 4489-4500, doi:10.1098/rsta.2008.0161, 2008.

Förster, M., Lühr, H., Reigber, C., König, R., Namgaladze, A. A., and Yurik, R. Y.: The CHAMP satellite and its space weather monitoring capability, in: Proceedings of the 14th ESA Symposium on European Rocket and Balloon Programmes and Related Research, SP-437, 255-259, Noordwijk, Netherland, 1999a.

Förster, M., Namgaladze, A. A., and Yurik, R. Y.: Thermospheric composition changes deduced from geomagnetic storm modeling, Geophys. Res. Lett., 26, 2625-2628, 1999b.

Förster, M., Paschmann, G., Haaland, S. E., Quinn, J. M., Torbert, R. B., McIlwain, C. E., Vaith, H., Puhl-Quinn, P. A., and Kletzing, C. A.: High-latitude plasma convection from Cluster EDI: Variances and solar wind correlations, Ann. Geophys., 25, 16911707, 2007, http://www.ann-geophys.net/25/1691/2007/.

Förster, M., Rentz, S., Köhler, W., Liu, H., and Haaland, S. E.: IMF dependence of high-latitude thermospheric wind pattern derived from CHAMP cross-track measurements, Ann. Geophys., 26, 1581-1595, 2008,

http://www.ann-geophys.net/26/1581/2008/.

Förster, M., Feldstein, Y. I., Haaland, S. E., Dremukhina, L. A., Gromova, L. I., and Levitin, A. E.: Magnetospheric convection from Cluster EDI measurements compared with the groundbased ionospheric convection model IZMEM, Ann. Geophys., 27, 3077-3087, 2009,

http://www.ann-geophys.net/27/3077/2009/.

Förster, M., Haaland, S. E., and Doornbos, E.: Thermospheric vorticity at high geomagnetic latitudes from CHAMP data and its IMF dependence, Ann. Geophys., 29, 181-186, 2011, http://www.ann-geophys.net/29/181/2011/.

Friis-Christensen, E., Lühr, H., and Hulot, G.: Swarm: A constellation to study the Earth's magnetic field, Earth Planets Space, 58, 351-358, 2006.
Friis-Christensen, E., Lühr, H., Hulot, G., Haagmans, R., and Purucker, M.: Geomagnetic Research From Space, EOS Trans. AGU, 90, 213, doi:10.1029/2009EO250002, 2009.

Haaland, S. E., Paschmann, G., and Sonnerup, B. U. Ö.: Comment on "A new interpretation of Weimer et al.'s solar wind propagation delay technique" by Bargatze et al., J. Geophys. Res., 111, A06102, doi:10.1029/2005JA011376, 2006.

Haaland, S. E., Paschmann, G., Förster, M., Quinn, J. M., Torbert, R. B., McIlwain, C. E., Vaith, H., Puhl-Quinn, P. A., and Kletzing, C. A.: High-latitude plasma convection from Cluster EDI measurements: Method and IMF-dependence, Ann. Geophys., 25, 239-253, 2007, http://www.ann-geophys.net/25/239/2007/.

Hardy, D. A., Gussenhoven, M. S., and Holeman, E. A.: A statistical model of auroral electron precipitation, J. Geophys. Res., 90, 4229-4248, 1985.

Häusler, K. and Lühr, H.: Nonmigrating tidal signals in the upper thermospheric zonal wind at equatorial latitudes as observed by CHAMP, Ann. Geophys., 27, 2643-2652, 2009,

http://www.ann-geophys.net/27/2643/2009/.

Hays, P. B., Killeen, T. L., Spencer, N. W., Wharton, L. E., Roble, R. G., Emery, B. A., Fuller-Rowell, T. J., Rees, D., Frank, L. A., and Craven, J. D.: Observations of the dynamics of the polar thermosphere, J. Geophys. Res., 89, 5597-5612, 1984.

Killeen, T. L. and Roble, R. G.: Thermosphere Dynamics: Contributions from the First 5 Years of the Dynamics Explorer Program, Rev. Geophys., 26, 329-367, 1988.

Killeen, T. L., Won, Y.-I., Niciejewski, R. J., and Burns, A. G.: Upper thermosphere winds and temperatures in the geomagnetic polar cap: Solar cycle, geomagnetic activity, and interplanetary magnetic field dependencies, J. Geophys. Res., 100, 21,32721,342, 1995.

Lühr, H., Rentz, S., Ritter, P., Liu, H., and Häusler, K.: Average thermospheric wind pattern over the polar regions, as observed by CHAMP, Ann. Geophys., 25, 1093-1101, 2007, http://www.ann-geophys.net/25/1093/2007/.

Lühr, H., Park, J., Ritter, P., and Liu, H.: In-situ CHAMP observation of ionosphere-thermosphere coupling, Space Sci. Rev., 168 237-260, doi:10.1007/s11214-011-9798-4, 2012.

Namgaladze, A. A., Korenkov, Y. N., Klimenko, V. V., Karpov, I. V., Bessarab, F. S., Surotkin, V. A., Glushchenko, T. A., and Naumova, N. M.: A global numerical model of the thermosphere, ionosphere, and protonosphere of the Earth, Geomagn. Aeron. (Engl. translation), 30, 515-521, 1990.

Namgaladze, A. A., Korenkov, Y. N., Klimenko, V. V., Karpov, I. V., Surotkin, V. A., and Naumova, N. M.: Numerical modelling of the thermosphere-ionosphere-protonosphere system, J. Atmos. Terr. Phys., 53, 1113-1124, 1991.

Namgaladze, A. A., Korenkov, Y. N., Klimenko, V. V., Karpov, I. V., Bessarab, F. S., Surotkin, V. A., Glushchenko, T. A., and Naumova, N. M.: Global model of the thermosphere-ionosphere-protonosphere system, Pure Appl. Geophys., 127, 219254, 1988.

Namgaladze, A. A., Martynenko, O. V., Namgaladze, A. N., Volkov, M. A., Korenkov, Y. N., Klimenko, V. V., Karpov, I. V., and Bessarab, F. S.: Numerical simulation of an ionospheric disturbance over EISCAT using a global ionospheric model, J. Atmos. Terr. Phys., 58, 297-306, 1996a.

Namgaladze, A. A., Namgaladze, A. N., and Volkov, M. A.: 
Numerical modelling of the thermospheric and ionospheric effects of magnetospheric processes in the cusp region, Ann. Geophys., 14, 1343-1355, 1996 b.

Namgaladze, A. A., Martynenko, O. V., and Namgaladze, A. N.: Global model of the upper atmosphere with variable latitudinal integration step, International Journal of Geomagnetism and Aeronomy, 1, 53-58, 1998a.

Namgaladze, A. A., Martynenko, O. V., Volkov, M. A., Namgaladze, A. N., and Yurik, R. Y.: High-latitude version of the global numerical model of the Earth's upper atmosphere, Proceedings of the Murmansk State Technical University, 1, 23-84, 1998b.

Namgaladze, A. A., Namgaladze, A. N., and Volkov, M. A.: Seasonal effects in the ionosphere-thermosphere response to the precipitation and field-aligned current variations in the cusp region, Ann. Geophys., 16, 1283-1298, 1998c.

Namgaladze, A. A., Förster, M., and Yurik, R. Y.: Analysis of the positive ionospheric response to a moderate geomagnetic storm using a global numerical model, Ann. Geophys., 18, 461-477, 2000 , http://www.ann-geophys.net/18/461/2000/.

Papitashvili, V. O., Christiansen, F., and Neubert, T.: A new model of field-aligned currents derived from high-precision satellite magnetic field data, Geophys. Res. Lett., 29, 1683, doi:10.1029/ 2001GL014207, 2002.

Rees, D. and Fuller-Rowell, T. J.: The response of the thermosphere and ionosphere to magnetospheric forcing, Phil. Trans. R. Soc. London, A328, 139-171, 1989.

Reigber, C., Lühr, H., and Schwintzer, P.: CHAMP mission status, Adv. Space Res., 30, 129-134, 2002.

Rentz, S. and Lühr, H.: Climatology of the cusp-related thermospheric mass density anomaly, as derived from CHAMP observations, Ann. Geophys., 26, 2807-2823, 2008, http://www.ann-geophys.net/26/2807/2008/.

Ridley, A. J., Gombosi, T. I., and De Zeeuw, D. L.: Ionospheric control of the magnetosphere: conductance, Ann. Geophys., 22, 567-584, 2004, http://www.ann-geophys.net/22/567/2004/.

Ridley, A. J.: Effects of seasonal changes in the ionospheric conductances on magnetospheric field-aligned currents, Geophys. Res. Lett., 34, L05101, doi:10.1029/2006GL028444, 2007.

Ridley, A. J., Gombosi, T. I., Sokolov, I. V., Tóth, G., and Welling, D. T.: Numerical considerations in simulating the global magnetosphere, Ann. Geophys., 28, 1589-1614, 2010, http://www.ann-geophys.net/28/1589/2010/.

Ruohoniemi, J. M. and Greenwald, R. A.: Dependencies of high-latitude plasma convection: Consideration of interplanetary magnetic field, seasonal, and universal time factors in statistical patterns, J. Geophys. Res., 110, A09204, doi:10.1029/ 2004JA010815, 2005.
Schlegel, K., Lühr, H., St.-Maurice, J.-P., Crowley, G., and Hackert, C.: Thermospheric density structures over the polar regions observed with CHAMP, Ann. Geophys., 23, 1659-1672, 2005, http://www.ann-geophys.net/23/1659/2005/.

Siscoe, G. L.: Global force balance of region 1 current system, J. Atmos. Sol.-Terr. Phys., 68, 2119-2126, 2006.

Siscoe, G. L. and Siebert, K. D.: Bimodal nature of solar windionosphere-thermosphere coupling, J. Atmos. Sol.-Terr. Phys., 68, 911-920, 2006.

Sofko, G. J., Greenwald, R. A., and Bristow, W. A.: Direct determination of large-scale magnetospheric field-aligned currents with SuperDARN, Geophys. Res. Lett., 22, 2041-2044, 1995.

Thayer, J. P. and Killeen, T. L.: Vorticity and divergence in the highlatitude upper thermosphere, Geophys. Res. Lett., 18, 701-704, 1991.

Tobiska, K. W., Bouwer, S. D., and Bowman, B. R.: The development of new solar indices for use in thermospheric density modeling, in: AIAA/AAS Astrodynamics Specialist Conference and Exhibition, American Institute of Aeronautics and Astronautics (AIAA), 21-24 Aug 2006, Keystone, Colorado, 2006.

Tsyganenko, N. A.: A model of the near magnetosphere with a dawn-dusk asymmetry 1. Mathematical structure, J. Geophys. Res., 107, 1179, doi:10.1029/2001JA000219, 2002a.

Tsyganenko, N. A.: A model of the near magnetosphere with a dawn-dusk asymmetry 2. Parameterization and fitting to observations, J. Geophys. Res., 107, 1176, doi:10.1029/2001JA000220, 2002b.

Vasyliunas, V. M.: The mechanical advantage of the magnetosphere: solar-wind-related forces in the magnetosphereionosphere-Earth system, Ann. Geophys., 25, 255-269, 2007, http://www.ann-geophys.net/25/255/2007/.

Volkov, M. A. and Namgaladze, A. A.: Models of field-aligned currents needful to simulate the substorm variations of the electric field and other parameters observed by EISCAT, Ann. Geophys., 14, 1356-1361, 1996, http://www.ann-geophys.net/14/1356/1996/.

Weimer, D. R.: Maps of ionospheric field-aligned currents as a function of the interplanetary magnetic field derived from Dynamics Explorer 2 data, J. Geophys. Res., 106, 12889-12902, 2001.

Weimer, D. R., Ober, D. M., Maynard, N. C., Collier, M. R., McComas, D. J., Ness, N. F., Smith, C. W., and Watermann, J.: Predicting interplanetary magnetic field (IMF) propagation delay times using the minimum variance technique, J. Geophys. Res., 108, 1026, doi:10.1029/2002JA009405, 2003.

Weimer, D. R.: Improved ionospheric electrodynamic models and application to calculating Joule heating rates, J. Geophys. Res., 110, A05306, doi:10.1029/2004JA010884, 2005. 